\title{
Impressions of force in visual perception of collision events: A test of the causal asymmetry hypothesis
}

\author{
Peter A. White \\ Cardiff University, Cardiff, Wales
}

\begin{abstract}
When two objects interact they exert equal and opposite forces on each other. According to the causal asymmetry hypothesis, however, when one object has been identified as causal and the other as that in which the effect occurs, the causal object is perceived as exerting greater force on the effect object than the latter is perceived as exerting on the former. An example of this is a stimulus in which one object moves toward another stationary one, and when contact occurs the former stops and the latter moves away. In this situation the initially moving object is identified as causal, so the causal asymmetry hypothesis predicts that more force will be judged to be exerted by the moving object on the stationary one than by the stationary one on the moving one. Participants' judgments consistently supported this hypothesis for a variety of stimuli in which kinematic parameters were varied, even when the initially moving object reversed direction after contact.
\end{abstract}

Imagine a stimulus involving two black rectangles or discs, A and B, against a white ground. B is stationary in the middle of the frame. A moves toward it in a straight line at constant speed. When A contacts B, B starts moving in the same direction at the same or slightly lower speed, and A stops. Observers of this stimulus reliably report a perceptual impression that A makes B move; in other words, they perceive B's motion as caused by A bumping into it. This perceptual impression was first reported by Michotte (1946), who called it "l'effet lancement," translated into English as the launching effect (Michotte, 1963), and many subsequent studies have confirmed it as a reliable feature of perception (Gordon, Day, \& Stecher, 1990; Scholl \& Tremoulet, 2000).

If there were real objects in this stimulus, the interaction between them would conform to Newton's third law of motion, according to which the force exerted by A on $\mathrm{B}$ is equal and opposite to the force exerted by B on A. However people do not perceive the launching effect stimulus in accordance with Newton's third law. I have argued (White, 2006) that the visual impression in the launching effect exhibits and exemplifies a general phenomenon in causal cognition called the causal asymmetry. According to this, once objects in an interaction have been assigned the roles of cause and effect, they are not perceived or understood as exerting equal and opposite forces on each other. Instead the cause object is seen as exerting more force than the effect object. There are two versions of this, one for each object. In the version of interest here, the role of the effect object's mass and velocity in determining the cause object's motion after contact is neglected. People do not see B as making A stop. Support for this claim consists of an absence of evidence for any report that observers see B making A stop (Gordon et al., 1990; Michotte, 1963; Schlottmann, Ray, Demetriou, \& Mitchell, 2006; White, 2006). There does not appear to have been any study in which participants were asked to report their impressions of the forces exerted by the objects. The present research is designed to test the causal asymmetry hypothesis by using a force judgment measure.

The causal asymmetry applies once the roles of cause and effect are assigned in an interaction. But it is not clear a priori how this is done. In the typical launching effect stimulus, why should A be assigned the role of cause and not B? White (2006) argued that this role assignment followed perception of activity prior to contact. Thus, in the launching effect stimulus $\mathrm{A}$ is active prior to contact and $\mathrm{B}$ is inactive, motionless. Because of this the active object, $A$, is then assigned the role of cause and the inactive object, B, that of effect. The causal asymmetry hypothesis then predicts that $\mathrm{A}$ will be judged to exert more force on $\mathrm{B}$ than B on A.

If this argument about causal role assignment is correct, then when both objects are active prior to contact there is no clear criterion for assigning the role of cause to one object and not the other. In this case the causal asymmetry would not be predicted to occur and the objects should be perceived to exert more nearly equal forces on each other. In particular, B should be judged to exert more force on A when B is moving prior to contact than when it is stationary. To test this reasoning, two sets of stimuli were employed in the present study, one set of stimuli in which 
B was motionless prior to contact and one in which B was moving. It is predicted that the causal asymmetry will be found in the former set but not in the latter, and in particular that B will generally be judged to exert more force on $A$ in the latter set than in the former.

\section{METHOD}

\section{Participants}

Thirty-two first-year undergraduate students of psychology participated in return for course credit. All had normal or corrected-tonormal vision.

\section{Apparatus, Stimuli, and Design}

Stimuli consisted of frame sequences generated by a Macintosh G3 computer and displayed on a Mitsubishi Diamond Plus 71 color monitor. The frames were presented in phase with the computer's vertical blank signal and appeared at the refresh rate of $75 \mathrm{~Hz}$. Each frame was 500 pixels $(18.5 \mathrm{~cm})$ wide $\times 300$ pixels $(11.1 \mathrm{~cm})$ high. The boundaries of the frame appeared on the screen as thin black lines. These disappeared between stimulus presentations, leaving the screen uniform white. All sequences consisted of 200 frames, each lasting $2.7 \mathrm{sec}$. The background of each frame was uniform white throughout. There were two sets of stimuli.

Stationary set. The term "stationary set" refers to the fact that one of the objects was stationary before contact. Stimuli were variations on the typical launching effect stimulus. One object (hereafter the right object) was initially stationary in the center of the frame. The other object (the left object) started at the left edge of the frame and moved horizontally toward the right object in a straight line at constant speed. ${ }^{1}$ When contact occurred the left object stopped moving and the right object started moving horizontally from left to right at constant speed, eventually leaving the frame at the right edge. Both objects were discs $1.48 \mathrm{~cm}$ in diameter. One was black and one was white, that is, a white disc with a black rim.

Three variables were manipulated in a within-subjects design. Speed of the left object before contact (left before) had three values, $8.1 \mathrm{~cm} / \mathrm{sec}$ (slow), $13.5 \mathrm{~cm} / \mathrm{sec}$ (medium), and $18.9 \mathrm{~cm} / \mathrm{sec}$ (fast). Motion of the left object after contact (left after) had three values, returning toward the left edge of the frame at $5.4 \mathrm{~cm} / \mathrm{sec}$ (left motion), remaining stationary at the point of contact (no motion), and moving to the right at $5.4 \mathrm{~cm} / \mathrm{sec}$ (right motion). Speed of the right object after contact (right after) had three values, $8.1 \mathrm{~cm} / \mathrm{sec}$ (slow), $13.5 \mathrm{~cm} / \mathrm{sec}$ (medium), and $18.9 \mathrm{~cm} / \mathrm{sec}$ (fast). These manipulations yielded a total of 27 stimuli.

Moving set. This set was termed the moving set because both objects were in motion before coming into contact. Stimuli were variations on a sequence in which both objects moved toward each other, came into contact, and then moved apart in the directions from which they had come. As in the stationary set, all movements were horizontal. The right object was initially positioned at the right edge of the frame, and the left object at the left edge, both halfway up. Both objects moved toward the center in a straight line at constant speed until they came into contact. Each object then returned in the direction from which it had come, in a straight line at constant speed, eventually leaving the frame. The objects were identical to those in the stationary set.

Four variables were manipulated, being the speeds of both objects before and after contact. Speed of the left object before contact (left before) had two values, $10.8 \mathrm{~cm} / \mathrm{sec}$ (slow) and $21.6 \mathrm{~cm} / \mathrm{sec}$ (fast). Speed of the right object before contact (right before) had the same two values. Speed of the left object after contact (left after) had two values, $5.4 \mathrm{~cm} / \mathrm{sec}$ (slow) and $10.8 \mathrm{~cm} / \mathrm{sec}$ (fast). Speed of the right object after contact (right after) had the same two values. These manipulations yielded a total of 16 stimuli.

Dependent measures. The measures were judgments of the amount of force exerted by each object on the other. Participants were asked, "How much force did the black circle exert on the white circle when they collided?" and "How much force did the white circle exert on the black circle when they collided?" Participants were instructed to answer by writing a number from 0 to 100 , where 0 means "no force at all" and 100 means "maximum possible force." They were instructed that they could put any number between 0 and 100.

Counterbalancing. Coloring of the two objects (black or white) was counterbalanced, and order of presentation of the two sets was counterbalanced orthogonally to this, with equal numbers of participants at each combination. These factors had no significant effects in any analysis and will not be further discussed.

\section{Procedure}

The experiment was run by an experimenter blind to the aims and hypotheses of the experiment. Participants were run individually in a small windowless laboratory, empty except for the equipment used for the experiment and with fluorescent lighting giving a low ambient light level. Participants were seated so that their faces were approximately $75 \mathrm{~cm}$ from the screen, and they were permitted to adjust this distance slightly for personal comfort. Participants first filled out an informed consent form. The experimenter introduced the experiment by giving the participant written instructions telling them that they would see stimuli involving collisions between two objects, and describing the force rating task.

The experimenter then told the participant that he would ask if they were ready. This was their cue to fixate the screen. When they said "yes," the first stimulus was presented. They then made their ratings in the appropriate place on a written set of rating scales. This procedure was re-iterated until all the stimuli in whichever set was presented first had been shown. The experimenter then proceeded to the other set and the same procedure was followed. The order of presentation of the stimuli in each set was randomized independently for each participant. Each stimulus was presented once only.

\section{RESULTS}

\section{Stationary Set}

Mean force ratings for the left object are depicted in Figure 1 and those for the right object in Figure 2. Figure 2 shows that force ratings for the right object were all low and in all cases lower than the corresponding ratings for the left object. This difference between ratings of the left and right objects was confirmed by 27 one-way ANOVAs comparing ratings of the two objects within each stimulus across participants. All were significant at $p<.001$, and the lowest $F$ ratio obtained was $22.41(d f=1,31$ in each analysis). There was therefore a strong and consistent tendency to give higher force ratings to the left object than to the right object. This tendency was found even when the left object reversed direction after contact.

The left object was rated significantly higher than the right object by 31 of the 32 participants. This consistency across individuals was confirmed by 32 one-way ANOVAs, comparing force ratings across stimuli for each participant. For 31 participants, $p<.001$, and the lowest $F$ ratio obtained was 39.78 ( $d f=1,26$ in each analysis). For one participant there was no significant difference between force ratings for the two objects $(F<1)$. This participant did not judge in accordance with Newton's third law, and appeared to be making random responses.

Looking at the independent variables, force ratings for the left object tended to rise as the speed of that object before contact increased, and they also tended to rise as the speed of the right object after contact increased. These 

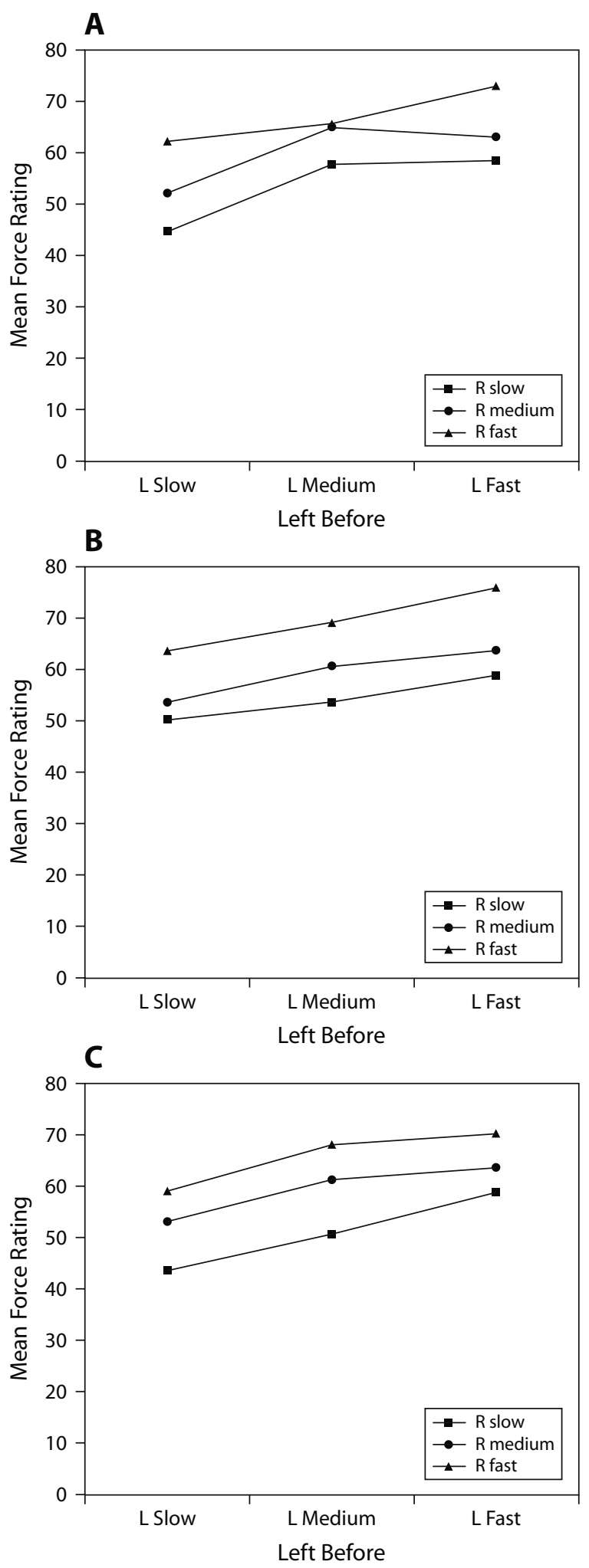

Figure 1. Mean force judgments for the left object in the stationary set. Individual panels show means for motion of the right object after contact (slow, medium, fast) at each speed of the left object before contact (slow, medium, and fast). (A) Left object moving to the left after contact. (B) Left object stationary after contact. (C) Left object moving to the right after contact. tendencies can be seen in the three panels of Figure 1, and were confirmed by three-way ANOVA with repeated measures. There was a significant main effect of left before $\left[F(2,62)=22.61, M S_{\mathrm{e}}=437.95, p<.001\right]$. Post hoc paired comparisons with the Newman-Keuls test revealed significant differences in all cases, with the order fast $(65.1)>$ medium $(61.3)>$ slow (53.6). There was a significant main effect of right after $[F(2,62)=44.29$, $\left.M S_{\mathrm{e}}=342.39, p<.001\right]$. Post hoc comparisons revealed significant differences in all cases, with the order fast (67.5) $>$ medium (59.6) $>$ slow (53.0). There were no other significant effects in this analysis.

Force ratings for the right object were influenced only by the behavior of the left object after contact, with higher ratings when the left object reversed direction than when it did not. This tendency can be seen in Figure 2. All of the means in panel A of this Figure are higher than all of the means in the other two panels. In the ANOVA for the right object, there was a significant effect of left after $[F(2,62)=$ $\left.22.02, M S_{\mathrm{e}}=724.80, p<.001\right]$. Post hoc comparisons revealed a higher mean at left motion (19.4) than at the other two values, which did not differ significantly (no motion, 7.3; right motion, 5.8). This main effect was qualified by a significant interaction with left before $[F(4,124)=2.67$, $\left.M S_{\mathrm{e}}=84.96, p<.05\right]$. Simple effects analyses revealed significant tendencies resembling the main effect of left after at all three values of left before. The interaction was only just statistically significant, and does not appear to indicate much effect of the left object's motion before contact. There were no other significant effects.

\section{Moving Set}

Mean force ratings for both objects are presented in Figure 3 . Mean ratings were consistently moderate, with no mean less than 33 or greater than 67 . Ratings of each object within each stimulus across participants were analyzed with 16 one-way ANOVAs. The results showed that, whenever one object moved faster than the other before contact, judgments of force were significantly greater for the faster object (eight results, lowest $F=10.66, d f 1,31$ in all cases, all $p<.01$ ). Whenever both objects moved at the same speed before contact there were no significant differences (eight results).

A 2 within (object: left vs. right) $\times 2$ within (left before: slow vs. fast) $\times 2$ within (right before: slow vs. fast) $\times 2$ within (left after: slow vs. fast) $\times 2$ within (right after: slow vs. fast) ANOVA was carried out. Because of the large number of effects in a five-way design, the Bonferroni correction was applied and the significance level was set at .002 .

Each panel of Figure 3 shows the same pattern of interaction. Force judgments were affected only by the behavior of the objects before contact. For each object, judgments were higher when it moved fast before contact than when it moved slowly before contact, and they were also higher when the other object moved slowly before contact than when it moved fast before contact. These tendencies can be seen in the crossover pattern in each of the four panels of Figure 3. These tendencies expressed 

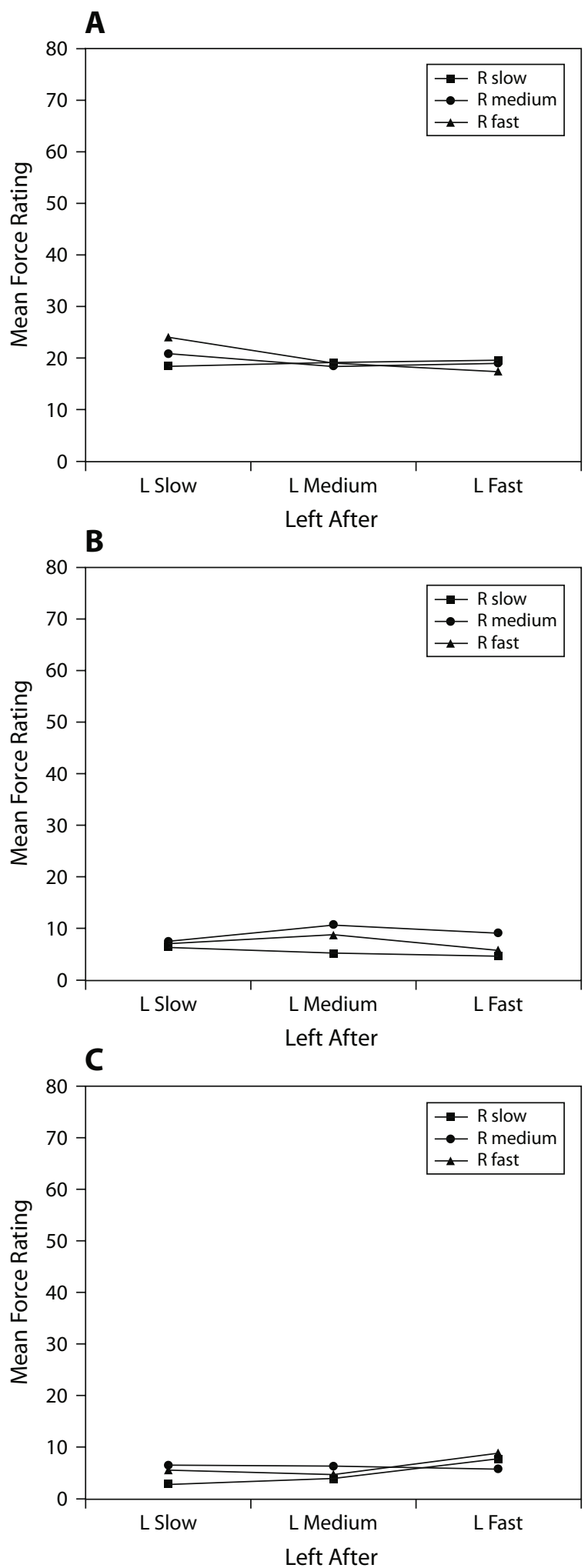

Figure 2. Mean force judgments for the right object in the stationary set. Individual panels show means for motion of the right object after contact (slow, medium, fast) at each speed of the left object before contact (slow, medium, and fast). (A) Left object moving to the left after contact. (B) Left object stationary after contact. (C) Left object moving to the right after contact. themselves in four significant effects, two main effects and two interactions. These effects will now be described in more detail.

There was a significant main effect of left before $\left[F(1,31)=24.90, M S_{\mathrm{e}}=184.83, p<.002\right]$, with a higher mean at fast (51.3) than at slow (47.1). This main effect was qualified by a significant interaction with object $\left[F(1,31)=57.18, M S_{\mathrm{e}}=585.11, p<.002\right]$. Simple effects analysis showed that judgments of force for the left object were higher when that object was moving fast before contact (57.3) than when it was moving slowly before contact (41.6) $\left[F(1,31)=67.77, M S_{\mathrm{e}}=463.89, p<\right.$ .002]. Judgments of force for the right object were higher when the left object was moving slowly before contact (52.6) than when it was moving fast before contact (45.4) $\left[F(1,31)=21.63, M S_{\mathrm{e}}=306.05, p<.002\right]$.

There was a significant main effect of right before $\left[F(1,31)=21.62, M S_{\mathrm{e}}=348.82, p<.002\right]$, with a higher mean at fast (51.9) than at slow (46.5). This main effect was qualified by a significant interaction with object $\left[F(1,31)=60.38, M S_{\mathrm{e}}=726.69, p<.002\right]$. Simple effects analysis showed that judgments of force for the left object were higher when the right object was moving slowly before contact (53.3) than when it was moving fast before contact $(45.6)[F(1,31)=12.22$, $\left.M S_{\mathrm{e}}=615.48, p<.002\right]$. Judgments of force for the right object were higher when it was moving fast before contact (58.2) than when it was moving slowly before contact (39.7) $\left[F(1,31)=95.43, M S_{\mathrm{e}}=460.03, p<\right.$ $.002]$. This interaction is the mirror image of the previous interaction.

\section{DISCUSSION}

The results supported the predictions derived from the causal asymmetry hypothesis. In all 27 stimuli in the stationary set significantly higher ratings were given to the left object than to the right object. All participants except one consistently gave higher ratings of force to the left object than to the right object.

Comparison between the two sets of stimuli is also appropriate. In the stationary set there were 9 stimuli in which the left object moved back toward the left after contacting the right object. The mean ratings of force exerted by the right object ranged from 17.2 to 23.9 on the 101-point scale. In the moving set there were 8 stimuli in which the left object moved back toward the left after contact, at the same speed as in the stationary set stimuli. The mean ratings of force exerted by the right object ranged from 33.1 to 60.9. Each of these means was therefore substantially higher than any of the 9 in the stationary set. The only difference between the stimuli was that the right object was in motion before contact in the moving set and stationary before contact in the stationary set. The findings therefore give strong support to the causal asymmetry hypothesis.

The causal asymmetry was proposed as a pervasive tendency to see the object assigned the role of cause as exerting more force on the object assigned the role of effect than vice versa. This tendency was found in the re- 

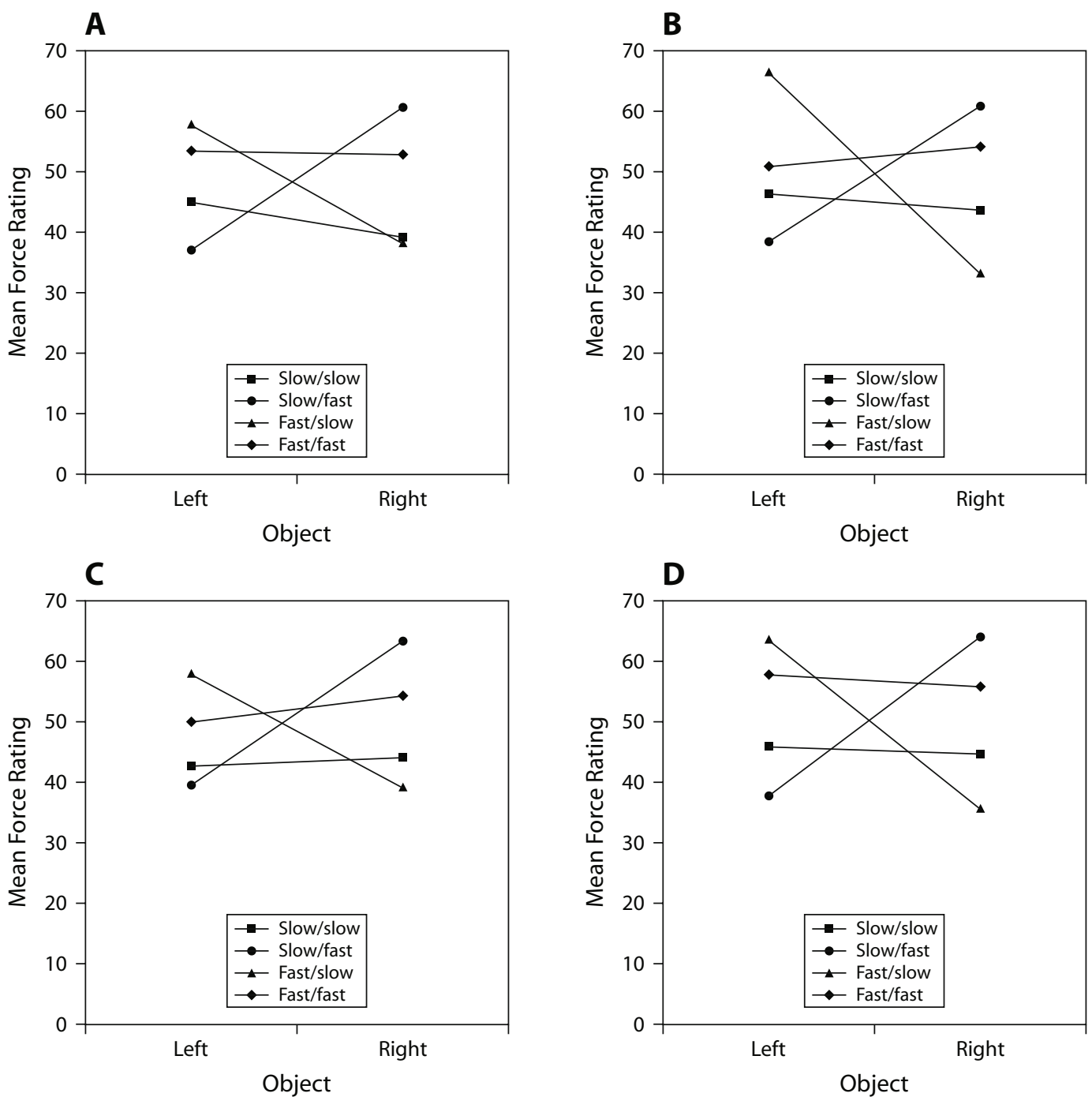

Figure 3. Mean force judgments for both objects in the moving set. Individual panels show means for all combinations of speeds of the objects before contact (the key identifies the speed of the left object first) at each combination of speeds of both objects after contact. (A) Both objects slow after contact. (B) Left object slow after contact, right object fast after contact. (C) Left object fast after contact, right object slow after contact. (D) Both objects fast after contact.

sults, but the causal asymmetry does not account for all significant variations in judgments of force. This is most evident for the moving set stimuli. In the moving set both objects were in motion before contact so there is no clear assignment of causal roles to one and not the other, so no causal asymmetry was predicted. The results were consistent with this prediction, but there were still consistent variations in force judgments across stimuli. For example, when the speed of the right object before contact was fast, judgments of force were greater for the right object and less for the left object than when the speed of the right object before contact was slow. Newton's third law applies to the moving set stimuli (or it would if they were real objects) but, even though the causal asymmetry does not apply to these stimuli, the objects were still not judged to be exerting equal forces on each other except when their speeds were equal.
Whatever may account for these variations in force judgments, it is not likely that it also accounts for the evidence for the causal asymmetry. The main reason for this contention is that the causal asymmetry is a domain general feature of causal cognition and not confined to perception. Evidence for it can be found in research on naive physics and semantics (White, 2006). As an example, diSessa (1982) set a task in which participants applied simulated pushes to change the direction of motion of a computergenerated object that behaved in accordance with Newton's laws of motion. The participants consistently neglected the causally relevant properties of the object, which resulted in predictable errors in the outcomes of their pushes. This is evidence of the causal asymmetry occurring in a different domain. The push is assigned the role of cause and the object that of effect, and the object's properties are then neglected in the choice of direction of the push. 
One possible alternative to the hypothesis that impressions of force are a perceptual phenomenon is that participants might be making ratings that depend on a narrow interpretation of the word "force" in the instructions. Thus, they might think that the right object is affecting the left object in some way but not interpret that as a case of force. This would lead them to give low ratings on the force measure. This interpretation is unlikely because studies by Talmy (1988) and Wolff and Song (2003) have shown that ideas of force constitute a generic concept that covers a variety of kinds of interactions between objects. Thus, if people judged that the right object was affecting the left object, this research implies that they would understand that influence as a kind of force. They should therefore be happy to use a force judgment measure as an indicator of the kind of influence they judge to be occurring. Whether the observed judgments reflect perception, cognitive processes, or linguistic processing cannot be definitely established with this data set, but the causal asymmetry is a feature of all three, so any of the three possibilities would be consistent with the causal asymmetry hypothesis.

The judgments of force obtained in this research offer a new perspective on research on phenomenal causality, the study of visual impressions of interactions between objects, such as the launching effect (Michotte, 1963; Scholl \& Tremoulet, 2000). One possibility is that the causal impression is just an impression that a certain amount of force is being exerted by one object on another. Then the occurrence of the impression would be reported whenever the perceived force surpassed some criterion. It cannot be quite as simple as that because different kinds of causal impressions have different qualitative characteristics. The qualitative appearance of pulling, for example, is different from that of launching, and furthermore its occurrence is influenced in different ways by manipulations of stimulus parameters (Michotte, 1963; Natsoulas, 1961; White \& Milne, 1997). Therefore, the force exerted could also be qualitatively specific: a pulling force when the pulling impression occurs, a launching force when the launching effect occurs, and so on. Causal impressions may therefore be combinations of a qualitative characteristic and an impression of a certain amount of force being exerted. From this perspective, findings that the launching effect is less likely to be reported under some conditions than under others, such as increasing deviation in the launched object's direction of motion from that of the launching object (Michotte, 1963) could be interpreted as indicating that less force is perceived.

This possibility appears to be contradicted by one feature of the results. In the stationary set there was a greater difference between judgments of the left object and the right object when the left object moved slowly before contact and the right object moved quickly after contact than when the left object moved quickly before contact and the right object moved slowly after contact. But the latter condition is usually associated with the occurrence of the launching effect, and the launching effect has been reported to be weak or absent under the former condition (Michotte, 1963; Natsoulas, 1961). Thus, a stronger causal asymmetry appears to occur in a condition where the launching effect is weak or absent. However Michotte (1963) reported that, when the right object moves faster than the left object, launching is replaced by a different kind of causal impression which he called triggering, an impression that A somehow triggered B's motion, perhaps by releasing energy from within B. This impression was confirmed in a later study by Natsoulas (1961). Causal impressions therefore occur under both conditions; only the qualitative characteristics of the impression change. There is therefore no contradiction in the results. The results do suggest, however, that the qualitative characteristics of the causal impression are not fully specified by the impression of force that occurs. Further research could shed useful light on the relationship between force impressions and qualitative aspects of the causal impression.

\section{AUTHOR NOTE}

I am grateful to Alan Milne for creating the software used to construct the stimuli, to Iain Hamlin for acting as experimenter, and to Lorraine Woods for assistance with the figures. Correspondence concerning this article should be addressed to P. A. White, School of Psychology, Cardiff University, Tower Building, Park Place, Cardiff CF10 3AT, Wales (e-mail: whitepa@cardiff.ac.uk).

\section{REFERENCES}

DISessa, A. A. (1982). Unlearning Aristotelian physics: A study of knowledge-based learning. Cognitive Science, 6, 37-75.

Gordon, I. E., DAY, R. H., \& STECHER, E. J. (1990). Perceived causality occurs with stroboscopic movement of one or both stimulus elements. Perception, 19, 17-20.

MichotTe, A. (1946). La perception de la causalité. Louvain: Institut Supérior de Philosophie.

Michotte, A. (1963). The perception of causality. New York: Basic Books.

Natsoulas, T. (1961). Principles of momentum and kinetic energy in the perception of causality. American Journal of Psychology, 74, 394-402.

Schlottmann, A., Ray, E., Demetriou, N., \& Mitchell, A. (2006). Perceived social and physical causality in animated motions: Spontaneous reports and ratings. Acta Psychologica, 123, 112-143.

Scholl, B. J., \& Nakayama, K. (2002). Causal capture: contextual effects on the perception of collision events. Psychological Science, 13, 493-498.

Scholl, B. J., \& Tremoulet, P. D. (2000). Perceptual causality and animacy. Trends in Cognitive Sciences, 4, 299-309.

TAlmy, L. (1988). Force dynamics in language. Cognitive Science, 12, 49-100.

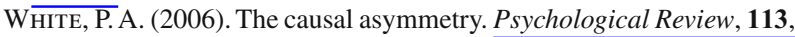
132-147.

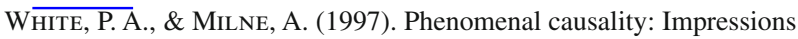
of pulling in the visual perception of objects in motion. American Journal of Psychology, 110, 573-602.

WOLFF, P., \& SonG, G. (2003). Models of causation and the semantics of causal verbs. Cognitive Psychology, 47, 276-332.

\section{NOTE}

1. Almost all studies of phenomenal causality have used left to right motion of the objects (e.g., Gordon et al., 1990; Michotte, 1963; Natsoulas, 1961), and I followed this convention. Studies of related phenomena have manipulated direction of motion and found no significant effect (Scholl \& Nakayama, 2002; White \& Milne, 1997), so it is not likely that direction would have a significant effect here.

(Manuscript received January 9, 2006; revision accepted for publication October 10, 2006.) 